\title{
Rumo a uma Plataforma Semântica de Conteúdos Educacionais Digitais: o Modelo Ontológico
}

\author{
João Carlos Gluz ${ }^{1}$, Rosa Maria Vicari ${ }^{2}$ \\ ${ }^{1}$ Programa de Pós-Graduação em Computação Aplicada (PIPCA) - Unisinos - São \\ Leopoldo - RS - Brazil \\ ${ }^{2}$ Centro Interdisciplinar de Tecnologias na Educação (CINTED) - Universidade Federal \\ do Rio Grande do Sul (UFRGS) - Porto Alegre - RS - Brazil \\ jcgluz@unisinos.br, rosa@inf.ufrgs.br
}

\begin{abstract}
The main hypothesis of the present work is that a learning object management platform, integrated with digital learning environments and able to recognize the semantics of its learning objects is the most appropriate content management system for educational purposes nowadays. Based on several years of accumulated experience working with these issues, this work proposes and evaluates an ontological model, which can represents the semantics of the several activities involved in the life-cycle of a learning object. A feasibility analysis of the proposed model and a comparative analysis of the properties of this model in respect to the state-of-the-art are also presented at the end of the paper.
\end{abstract}

\section{Introdução}

As Tecnologias de Informação e Comunicação (TIC) tem oportunizado mudanças nas relações entre as competências intelectuais e os conhecimentos. A aplicação das TIC na Educação, em particular, tem possibilitado novos paradigmas para situações de aprendizagem. Hoje há um amplo cenário de aplicação dessas tecnologias para fins educacionais, incluindo desde conteúdos educacionais digitais, passando por Ambientes Virtuais de Aprendizagem (AVA) e chegando a materiais animados, ferramentas de simulação educacional e jogos educativos. Conteúdos digitais educacionais e AVA passaram a ser vistos como tecnologias essenciais para a condução dos processos educacionais, sejam eles presenciais ou a distância. Um aspecto importante dos conteúdos educacionais digitais é que eles sejam padronizados para facilitar sua localização, reusabilidade e adaptabilidade. A tecnologia de Objetos de Aprendizagem (OA) se propõe a atender estas premissas, sendo um veículo importante para a ampla disseminação dos conteúdos digitais. Essa tecnologia fundamenta-se na hipótese de que é possível criar componentes de materiais educacionais em formato padronizado e organizá-los de forma a possibilitar sua reutilização [Wiley, 2001], tanto na produção de cursos on-line, quanto na sua utilização como parte de uma aula presencial.

Em separado, as tecnologias de AVA e OA são fundamentais para os processos educacionais mediados por computador. Utilizados em conjunto, ambas tecnologias podem oferecer muito mais, potencializando uma sinergia entre conteúdos educacionais padronizados e ambientes ricos em ferramentas de interação e apoio ao ensino. Além disso, se os ambientes educacionais forem capazes de reconhecer, mesmo que parcialmente, o significado (semântica) dos termos e conceitos representados nos OA, então muitas possibilidades se abrem para facilitar as atividades dos usuários do ambiente. O suporte a atividades como autoria de conteúdos educacionais, uso destes conteúdos na mediação de processos de aprendizagem, além do acompanhamento e avaliação destes processos, poderia se centrar muito mais na terminologia e nos aspectos da área de aplicação (Educação), com muito menos necessidade de 
conhecimentos técnicos de Informática para sua consecução.

Este é a hipótese centra de pesquisa deste trabalho: uma plataforma de gerência de conteúdos digitais organizados na forma de OA, devidamente integrada a um ambiente de ensino e capaz de reconhecer a semântica dos conteúdos e informações armazenados nos OA é o tipo de plataforma de conteúdos mais apropriado atualmente para a oferta de serviços, cursos e aplicações educacionais. Nesse sentido, é proposto e avaliado um modelo ontológico para a representação da semântica dos OA, com base na análise e síntese dos principais resultados obtidos no projeto OBAA-MILOS [Gluz et al., 2012; 2010] em relação ao uso de ontologias para representação e gerência de OA.

\section{Estado da Arte}

O quadro geral das pesquisas envolvendo tratamento semântico de OA ou que busquem integrar AVA e OA é bastante díspar até o momento. O uso de ontologias para trazer semântica aos ambientes educacionais é um fenômeno atual (Isotani, Misoguchi et al., 2008). Tal tecnologia tem se mostrado útil na concepção de vários ambientes educacionais [Isotani, Isotani et al., 2008; Silva et al., 2009; Bittencourt et al., 2008; Hayashi, et al. 2009]. Ontologias são empregadas para definir as propriedades das entidades de um ambiente educacional, com uma tendência em dividir as ontologias educacionais em três tipos [Silva et al., 2009]: a) representação de domínios de ensino, b) representação de métodos e estratégias pedagógicas, e c) modelos de aluno. Também existem propostas que integram aspectos parciais desses três tipos [Mizoguchi et al., 2007; Hayashi et al., 2009]. Mais recentemente foram propostos modelos ontológicos [Bittencourt et al., 2009] voltados à representação de estruturas curriculares, modelos de aluno e aplicações educacionais.

A aplicação de ontologias para representação e tratamento semântico de OA é mais limitada. Em termos técnicos, muito do trabalho relacionado aos OA tem se centrado na criação de ferramentas que auxiliem no processo de autoria de OA, com destaque para ferramentas como FreeLoms (www.freeloms.org), eXe Learning [Barbone e Rifon, 2009] e Xerte (Ball e Tenney, 2008). Outra linha de trabalho se relaciona com a criação de repositórios que permitam a edição, armazenamento e recuperação de metadados de OA. Nessa linha se destacam sistemas como o DSPACE [Smith et al., 2003] para OA com metadados DCMI e FEB [Schreiner et al, 2012] que suporta metadados OBAA. Nenhum desses trabalhos utiliza tecnologias para o tratamento da semântica do OA. O suporte e integração de OA com AVA é uma frente de pesquisa ainda em estágio inicial. De acordo com [Penteado et al, 2014] esta integração é geralmente limitada a disponibilização de OA na forma de conteúdos "empacotados" de acordo com os padrões SCORM ou IMS-CP.

\section{Metodologia}

A metodologia de pesquisa deste trabalho percorre três movimentos principais: análise, síntese e avaliação. Inicialmente é feita uma análise dos principais resultados alcançados pelo projeto OBAA-MILOS [Gluz et al., 2012; 2010] na representação da semântica dos OA. Esta análise fundamenta a síntese de um modelo ontológico integrado que serve de base a uma plataforma semântica de serviços de OA. O fío condutor do processo de análise é baseado no ciclo de vida de OA quando analisado no contexto de um cenário de uso que integra um AVA hipotético com a plataforma semântica de serviços semânticos de OA. Os aspectos socioculturais do cenário, dirigem $\mathrm{o}$ processo de análise. A síntese se fundamenta na generalização das ontologias e mecanismos de interação obtidos com o projeto OBAA-MILOS, quando confrontados com os requisitos elicitados previamente na análise de cenário. A avaliação do modelo ontológico é feita de duas formas: (a) avaliação da viabilidade do modelo ontológico proposto neste trabalho através da análise e interpretação dos resultados dos experimentos realizados com ontologias no projeto OBAA-MILOS e (b) análise 
comparativa das propriedades do modelo ontológico proposto neste trabalho, incluindo implicações para os serviços de OA, em relação ao estado da arte.

\section{Premissas da Análise}

Uma lição importante aprendidas com o projeto OBAA-MILOS é a necessidade de considerar a perspectiva epistêmica na representação e tratamento dos OA [Gluz e Vicari, 2010]. Como consequência, assume-se como premissa epistêmica fundamental que os OA devem ser tratados desde o início como Objetos de Conhecimento (OC). Essa classificação não é arbitrária e pode ser contextualizada em relação a classificações existentes para os OA [Gomes et al., 2009] em uma posição intermediária, cobrindo uma área mais ampla do que os objetos inteligentes, mas ainda ficando inserida no contexto dos OA funcionais. A visão de um OA como OC é consistente com os objetivos dos OA nos contextos de ensino: espera-se que um OA seja criado por alguém com conhecimento a respeito de um domínio de ensino e que através do seu uso, outras pessoas (alunos) passem por um processo de internalização de conhecimentos deste domínio. Na verdade, o problema mais importante com essa visão é mais de ordem tecnológica do que educacional. Uma tecnologia capaz de reconhecer e representar, nem que seja parte do conhecimento existente em um OA, poderia ser muito útil, ajudando alunos e professores a melhor aproveitá-lo ou reusá-lo, auxiliando designers a projetar OA mais apropriados para as necessidades didáticas e pedagógicas dos seus usuários e facilitando o processo geral de catalogação, gerência e disseminação dos OA.

Outra lição importante aprendida no projeto OBAA-MILOS é que os OA não podem ser considerados apenas como um artefato tecnológico isolado, exclusivamente vinculado a apenas um usuário, mas devem ser considerados sob uma ótica social e cultural [Gluz et al., 2014b]. Neste trabalho tomamos como base para essa visão sociocultural a Teoria Sócio-Histórica de Vygotsky (1988), considerando-a como a perspectiva apropriada para considerar a forma como um OA poderia, idealmente, ser utilizado em processos de ensino. Essa abordagem também é consistente com a visão de OA como OC. Nesse caso o OA pode ser visto como um instrumento usado pelas ações de mediação que compõe o processo de mediação de conhecimento entre sujeitos em interação [Wertsch, 1998]. Aqui o OA é visto como um objeto que faz parte de uma cena de atenção conjunta entre ambos os sujeitos [Tomasello, 2001], sendo o objeto social da atenção de ambos que irá permitir a apropriação de novos conceitos. Nessa perspectiva um OA é um artefato sociocultural que serve como instrumento para a mediação deste processo. Essa visão se alinha com as principais características deste tipo de artefato: OA, como outros tipos de aplicações e ferramentas de software, são construções tecnológicas cujo valor e uso é determinado por normas culturais e sociais, que tais artefatos não apenas incorporam, mas ajudam a perpetrar. Na perspectiva dos seus usuários, mais que trazer um determinado conhecimento para um usuário individual, o OA pode se tornar um meio ou veículo de compartilhamento de conhecimentos entre os atores de um processo de ensino. Os OA, em conjunto com suas tecnologias de suporte, poderiam ser consideradas tecnologias sociais e ferramentas mentais ("minds tools") ao agir mais sobre os próprios sujeitos que sobre objetos [Jonassen, 1999]. Tais artefatos tecnológicos irão se constituir em signos, do ponto de vista sócio-histórico [Vygotsky, 1988], na medida em que permitem estruturar e organizar a ação humana como produtos e produtores da cultura numa dialética entre pessoas, sociedade, tecnologia e cultura.

\section{Ciclo de Vida de um OA}

Embora não seja uma abordagem usual da literatura sobre OA [Penteado et al., 2014], a experiência acumulada no projeto OBAA-MILOS [Gluz et al., 2012] indicou que na 
concepção de uma plataforma semântica de serviços para estes tipo de objetos deve-se considerar o ciclo de vida de um OA como unidade de análise elementar dos serviços a serem prestados aos OA. Nesse trabalho propomos que o ciclo de vida de um OA possa ser analisado nas fases mais gerais apresentadas na Tabela 1, já subdivididas nas atividades mais importantes de cada fase.

Tabela 1. Fases do Ciclo de Vida de um OA

\begin{tabular}{|l|l|}
\hline \multicolumn{1}{|c|}{ Fase } & \multicolumn{1}{c|}{ Atividade } \\
\hline Autoria do OA & $*$ Criação/edição do conteúdo \\
& $*$ Encapsulamento/empacotamento \\
& $*$ Criação/edição dos metadados \\
\hline Uso do OA & $*$ Busca e localização do objeto apropriado \\
& $*$ Disponibilização e apoio ao uso (alunos) \\
& $*$ Acompanhamento do uso (professores) \\
\hline \multirow{3}{*}{ Manutenção do OA } & $*$ Gerência de conteúdos/metadados \\
& $*$ Gerência de versões \\
& $*$ Adaptações para múltiplas plataformas \\
& $*$ Adaptações para requisitos acessibilidade \\
\hline
\end{tabular}

Essas fases não são muito distintas de outros tipos de artefatos tecnológicos, como aplicações ou ferramentas de software. Porém, existem algumas diferenças importantes: (a) a divisão dos OA em nível de conteúdos e nível de metadados, usada nas diversas padronizações de OA (IEEE-LOM, DublinCore, OBAA), leva a atividades distintas para cada nível e (b) a interoperação em múltiplas plataformas digitais (Web, tablets, TV Digital) ou o suporte à acessibilidade são funcionalidades importantes o suficiente para serem considerados em seu ciclo de vida.

\section{Técnica de Análise}

A análise será baseada na técnica $I^{*}$ de análise de sistemas sociais [Yu et al, 2011], que é uma ferramenta de análise (framework) orientada para modelar "ambientes organizacionais", caracterizados como sistemas sociais (instituições, organizações, empresas) com objetivos gerais, no qual os atores sociais estão comprometidos em trabalhar cooperativamente para atingi-los. Diagramas $I^{*}$ permitem especificar as relações sociais de dependência existentes entre os atores, além de representar as motivações e aspectos de intencionalidade dos atores.

Apesar de útil, a técnica $I^{*}$ possui importantes limitações quando se pretende incorporar aspectos culturais das relações sociais de dependência, algo que assumimos como necessário para a completa caracterização de um OA com artefato sociocultural. A cultura é uma parte importante de qualquer sociedade ou sistema social, porém a definição deste conceito abrange um grande contínuo de opções. Neste trabalho assumimos uma acepção de cultura mais vinculada a estudos antropológicos, usando uma das acepções do dicionário Aurélio (2004) para este conceito, onde cultura é definida como "o conjunto complexo dos códigos e padrões que regulam a ação humana individual e coletiva, tal como se desenvolvem em uma sociedade ou grupo específico, e que se manifestam em praticamente todos os aspectos da vida: modos de sobrevivência, normas de comportamento, crenças, instituições, valores espirituais, criações materiais, etc.". No contexto limitado de análise social oferecido pela ferramenta $I^{*}$, a cultura se concretiza nos significados (semântica) dos objetivos, tarefas e recursos compartilhados nas relações de dependência, e na forma (sintaxe) como estes elementos podem se expressos e comunicados de um ator para outro. Dessa maneira, cultura aqui assume um significado preciso, sendo definida pela linguagem e protocolos utilizados nas interações sociais derivadas ou implicadas pelas relações de dependência, 
e no significado (ontologia) atribuído aos termos dessa linguagem.

Em termos computacionais propomos uma extensão de $I^{*}$, assumindo que a cultura em um modelo $I^{*}$ pode incluir ontologias, linguagens e processos de interação subjacentes ao modelo. Uma cultura particular é representada pela diagramação de um retângulo de cantos arredondados, desenhado $s o b$ os elementos do diagrama englobados por essa cultura. Um diagrama pode fazer uso de mais de uma cultura, nesse caso os retângulos correspondente podem ser independentes ou podem se sobrepor, indicando, respectivamente, culturas (e, portanto, ontologias, linguagens e protocolos) não relacionadas entre si, ou culturas com elementos comuns. Elementos, sejam eles atores, objetivos, tarefas ou recursos, em áreas onde existem duas ou mais culturas sobrepostas terão significados equivalentes nas culturas envolvidas.

\section{Análise do Cenário de Uso}

Uma plataforma de conteúdos educacionais digitais pode ser utilizada de várias formas distintas, tanto como uma ferramenta independente ("standalone") para gerenciar este tipo de conteúdo, quanto integrada a um AVA. Embora o uso como ferramenta independente possa ser interessante em diversos casos, atualmente os AVA são, pelo menos no contexto da educação formal, a interface de utilização mais amplamente disseminada para acesso a material educacional [Penteado et al, 2014]. Assim no presente cenário iremos considerar a utilização integrada de plataforma semântica de conteúdos a um (hipotético) AVA. A Figura 1 resume os resultados de uma análise dessa situação voltada para a identificação dos principais atores neste cenário e seus respectivos objetivos, tarefas e recursos compartilhados.

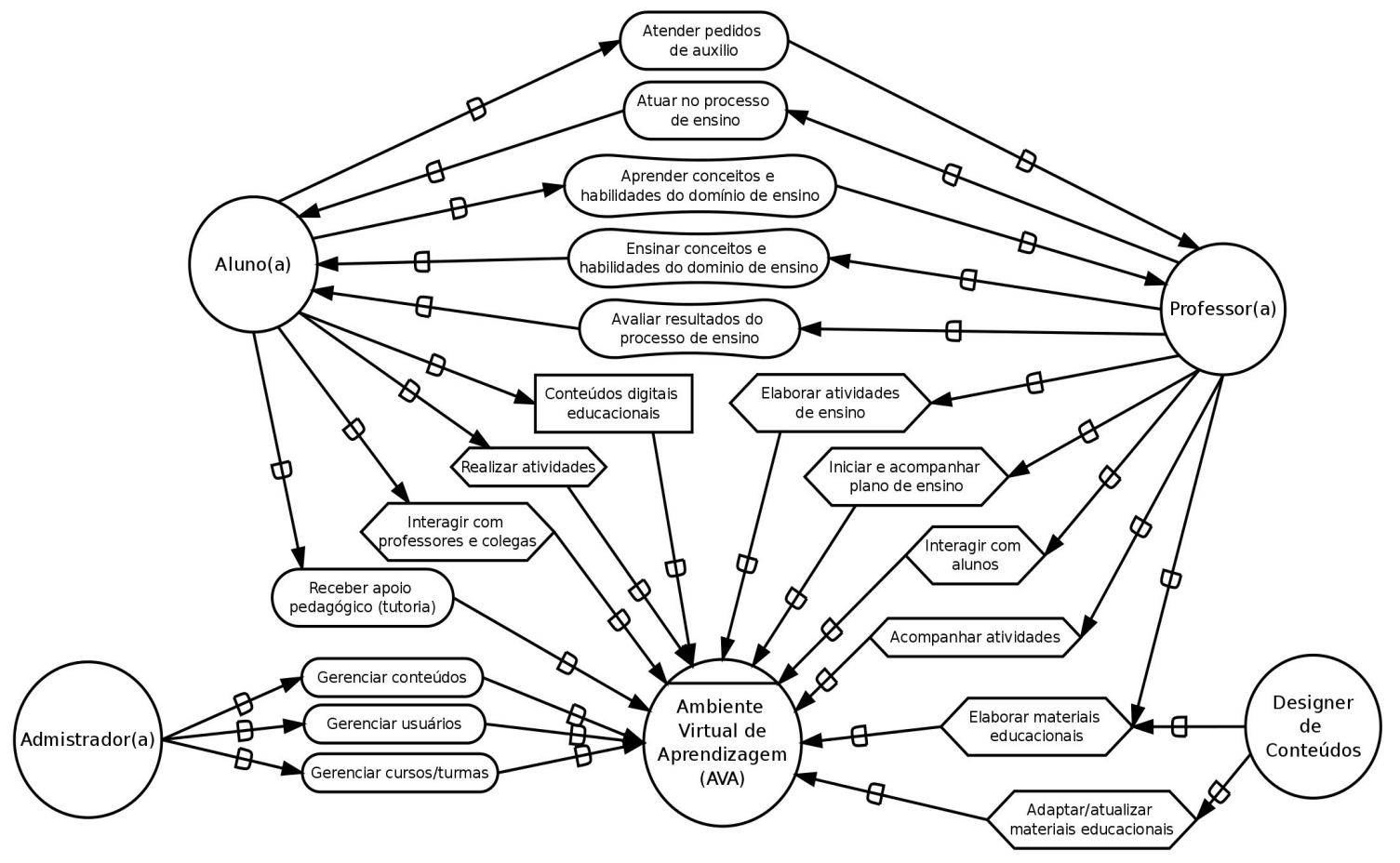

Figura 1. Cenário de Uso de AVA

O diagrama da Figura 1 é um diagrama de dependências estratégicas (modelo SD) de $I^{*}$ [Yu et al., 2011]. Nestes diagramas, círculos (p.ex. "Professor") representam atores de uma sistema social e círculos com uma barra horizontal na parte superior são especializações de atores na forma de agentes computacionais (p.ex. "AVA"). Atores e agentes realizam ações para atingir objetivos no ambiente, estabelecendo relações de dependência com outros atores para atingir esses objetivos. Essas dependências podem ser motivadas por objetivos concretos (retângulos ovalados), por tarefas ou atividades (hexágonos), por recursos (retângulos), ou por objetivos "soft" ou não-funcionais (ovais 
com reentrâncias). O modelo SD da Figura 1 elicita um conjunto mínimo de relações entre professores e alunos, mas que permitem identificar os principais impactos do AVA no processo de ensino. Certamente que outras relações sociais podem ser consideradas, mas aqui o foco se restringiu aos elementos influenciados ou impactados quando a mediação digital por meio do AVA é introduzida. Deve-se ressaltar que este modelo não pretende ser um modelo completo das relações sociais deste tipo de cenário, mas apenas um modelo apropriado para conduzir a análise do uso de conteúdos digitais no contexto da mediação tecnológica.

O modelo da Figura 1 propõe como objetivos não-funcionais do processo de ensino: a dependência que o aluno tem do professor para aprender os conceitos e habilidades no domínio de ensino, considerando o professor como parceiro mais experiente na mediação [Vygotsky, 1998] e as dependências que o professor tem em relação aos seus alunos para atingir suas metas de ensinar conceitos e habilidades, além de avaliar os resultados deste processo. São dois objetivos concretos do modelo: o aluno depende do atendimento do professor quando solicita auxílio para resolver dúvidas, e o professor depende do aluno para atuar no processo de ensino. O modelo assume que tais dependências são fundamentais para o processo de ensino e de responsabilidade dos atores humanos deste processo. O AVA entra como um instrumento de apoio a mediação digital, fornecendo ferramentas que permitam realizar uma série de tarefas, anteriormente realizadas presencialmente. A realização de atividades didáticopedagógicas, interação professor-aluno (ou entre colegas), elaboração de material educacional, entre outras atividades, agora são feitas por meio do AVA. Quando o AVA entra em cena, outros atores aparecem, incluindo a figura do administrador do AVA e também do designer/projetista de conteúdos digitais (que pode até ser um papel especializado dos professores).

O diagrama da Figura 2 estende essa análise preliminar, ilustrando quais objetivos, tarefas e recursos comuns são afetados quando o AVA é integrado a uma plataforma semântica de conteúdos digitais.

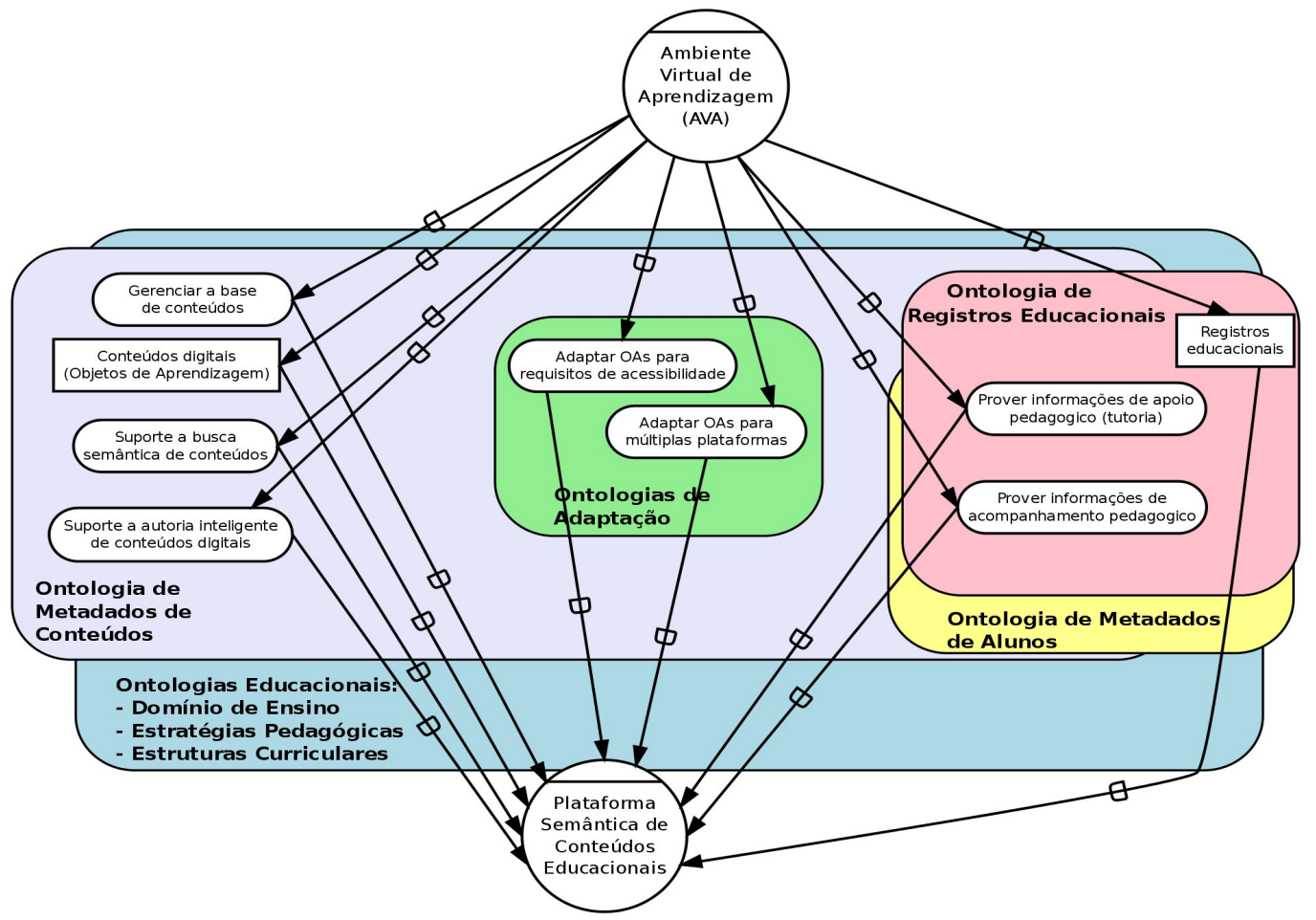

Figura 2. Cenário de Integração AVA x Plataforma Semântica de Conteúdos

Este diagrama identifica os principais objetivos e recursos compartilhados entre um AVA hipotético e uma plataforma de conteúdos capaz de reconhecer a semântica destes conteúdos. Nesse trabalho estamos supondo que é importante delimitar qual a 
semântica eventualmente assumida pelas interações sociais subjacentes às relações de dependência. Assim utilizamos a extensão de I* proposta na Seção 6 incorporando o contexto semântico na forma das ontologias subjacentes as interações sociais causadas pelas relações de dependência. Tais ontologias são representadas no diagrama SD através de retângulos maiores coloridos desenhados sob as relações de dependência. $\mathrm{O}$ contexto semântico de uma relação de dependência é definido pelas ontologias situadas sob o símbolo da relação. Embora não necessariamente completos, os objetivos e recursos elicitados na Figura 2 são coerentes com as etapas e atividades do ciclo de vida de um OA apresentadas na Seção 5. Gerenciar a base de conteúdos, assim como adaptar OA para requisitos de acessibilidade e para múltiplas plataformas são parte da etapa de manutenção do OA. O fornecimento dos OA como recursos e o suporte inteligente (sensível ao contexto semântico) ao processo de autoria, fazem para da etapa de autoria. Por fim, prover informações de apoio pedagógico (tutoria) e de acompanhamento do processo de ensino, sensíveis ao contexto desse processo, assim como oferecer um serviços de busca semântica de conteúdos, fazem parte da etapa de uso do OA.

\section{Estrutura do Modelo Ontológico}

As ontologias identificadas na Figura 2 são importantes porque fornecem a própria semântica da plataforma "semântica". Tais ontologias definem os contextos de ensino que serão usados na autoria ou busca de OA apropriados para um tópico de ensino. Todos esses tipos de ontologias foram pesquisados, concebidos e aplicados no decorrer do projeto OBAA-MILOS, mostrando quão úteis tais ontologias podem ser para a concepção e desenvolvimento de serviços inteligentes, capazes de reconhecer a semântica das diversas atividades envolvidas no ciclo de vida de um OA. Como resultado dessa pesquisa foi possível estabelecer um quadro geral para o modelo ontológico desse tipo de plataforma de serviços, apresentado na Figura $3^{\mathrm{f}}$.
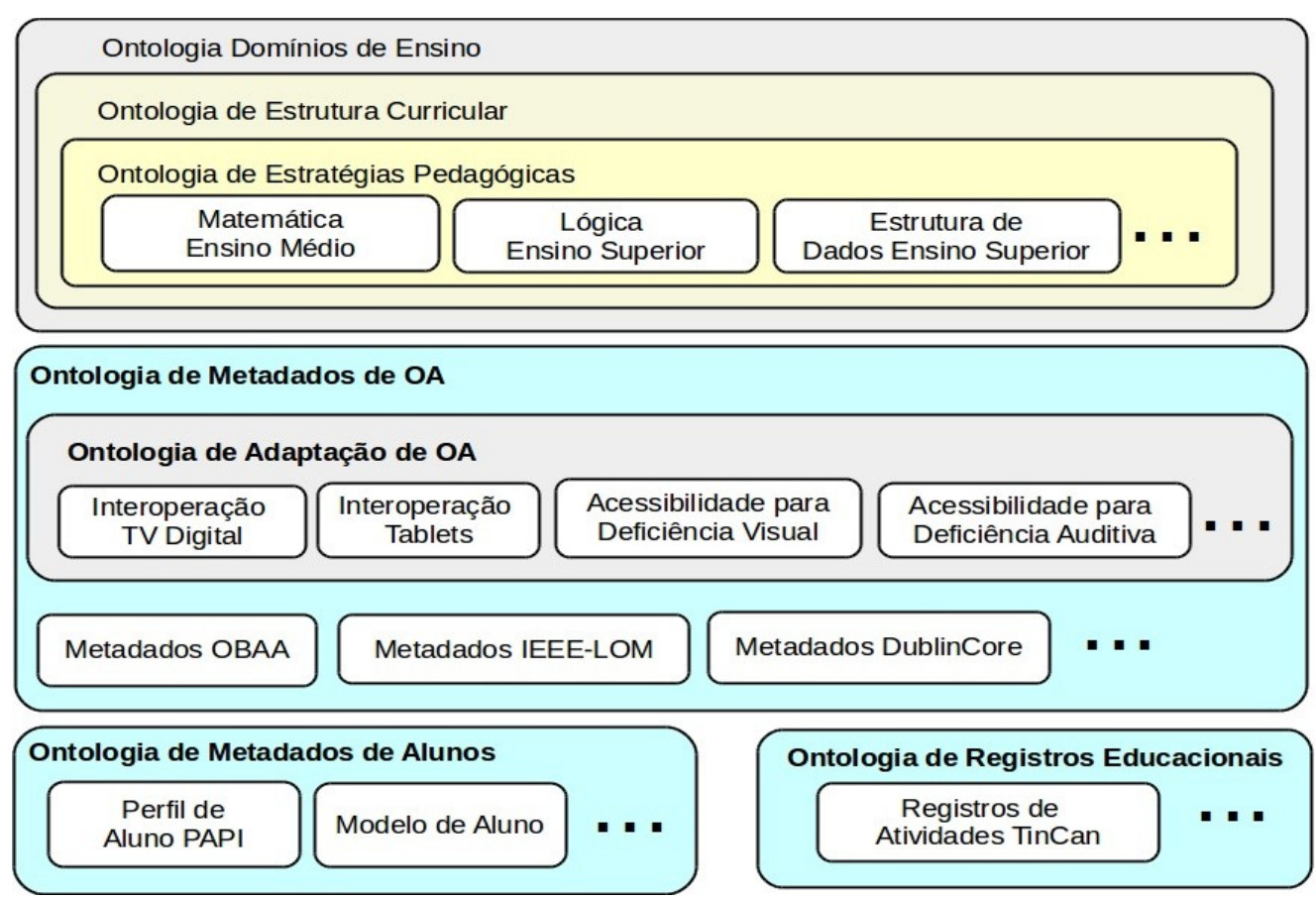

Figura 3. Visão Geral do Modelo Ontológico

Neste modelo as ontologias educacionais, pressupostas no diagrama da Figura 2, se estruturam em níveis, começando com a definição dos elementos do domínio de ensino, passando pela estrutura curricular de disciplinas sobre este domínio e eventualmente chegando na definição de estratégias e métodos pedagógicos para o ensino desta disciplina. A Figura 4 mostra como este processo ocorre. As ontologias de

1 Todas estas ontologias OWL estão disponíveis no site http://obaa.unisinos.br 
domínio de ensino, estrutura curricular e estratégias pedagógicas, cujo nível mais alto é apresentado na parte superior da Figura 4, são incluídas em uma ontologia de Matemática para o Ensino Médio, apresentada na parte inferior desta Figura. Processos similares de fusão de ontologias por inclusão permitem a integrar os conceitos e axiomas das ontologias de metadados e de adaptação de OA.

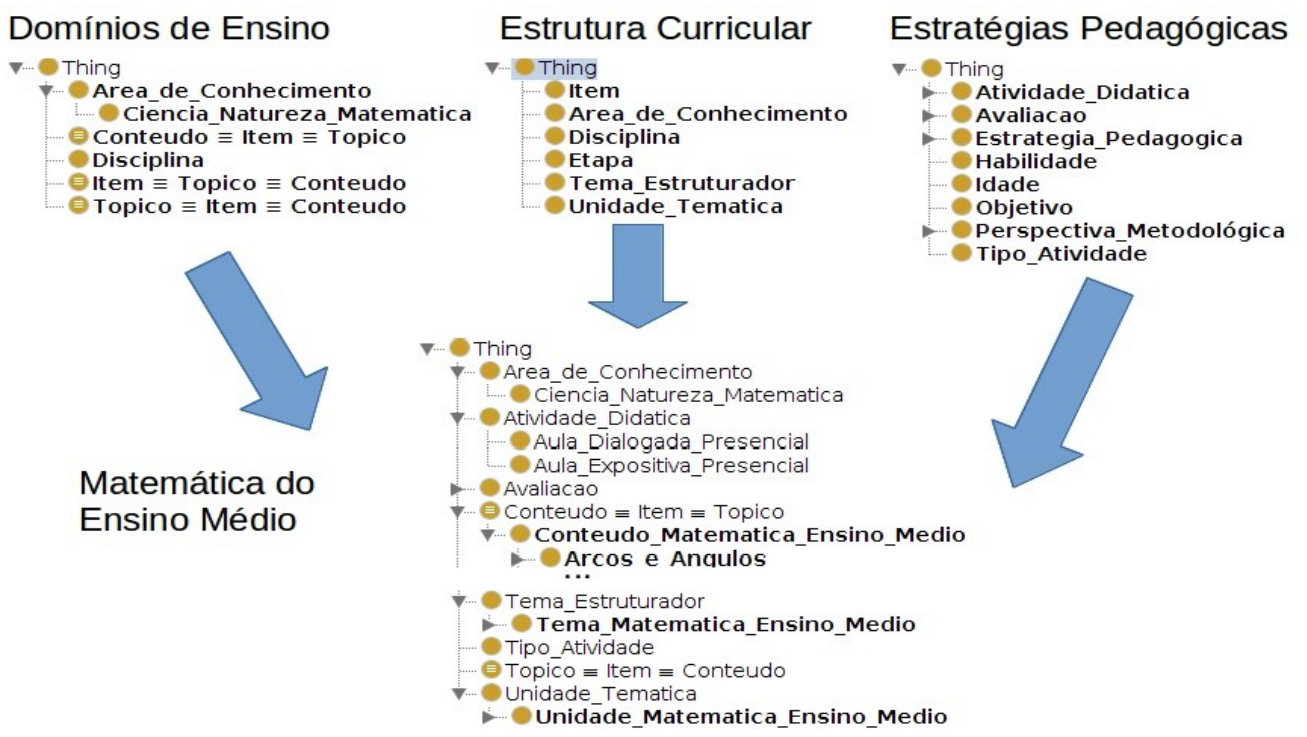

Figura 4. Fusão de Ontologias Educacionais

\section{Fusão, Alinhamento e Mapeamento de Ontologias}

A forma de fusão de ontologias por inclusão torna a utilização das ontologias mais prática, quando se precisa efetuar a busca de semântica de ỎA ou o preenchimento automático de metadados. Porém tal tipo de fusão necessita de uma certa padronização ou acordo na forma e estrutura das ontologias educacionais. A padronização de ontologias educacionais pode ser útil, mas certamente é um objetivo complexo de ser alcançado. Afortunadamente, existem técnicas que tornam este processo mais fácil, estabelecimento de forma automática (ou semiautomática) relações entre ontologias educacionais distintas. O alinhamento de ontologias é um dos mecanismos mais importantes para a integração de ontologias heterogêneas [Ehrig, 2007; Euzenat e Shvaiko, 2007]. Utilizando técnicas de alinhamento é possível incorporar e compatibilizar de forma gradativa um grande conjunto das ontologias educacionais, sem a necessidade de retrabalho ou refatoração completa destas ontologias. Apesar disso, tanto a fusão por inclusão quanto por alinhamento, não resolve a diferença existente entre ontologias de metadados e ontologias de conceitos educacionais. Ontologias educacionais representam conhecimentos e conceitos sobre conteúdos educacionais e métodos de ensino e aprendizagem, enquanto que ontologias de metadados representam tipos de dados e valores possíveis para os metadados. Pode-se supor que valores de metadados educacionais, em uma ontologia de metadados, possam ser associados à classes ou relacionamentos que representam conceitos educacionais, em uma ontologia educacional, porém tais entidades estão em níveis conceituais distintos, impossibilitando o uso de técnicas de alinhamento. Neste caso o problema de fusão de ontologias se reduz a um problema de mapeamento linguístico, que visa identificar as correspondências entre termos linguísticos que podem aparecer como valores dos metadados e os conceitos das ontologias educacionais.

\section{Avaliação do Viabilidade do Modelo Ontológico}

O modelo ontológico apresentado na Seção 8 sintetiza a experiência acumulada no decorrer do projeto OBAA-MILOS na modelagem e aplicação concreta de várias ontologias na construção de protótipos de ferramentas e serviços inteligentes de suporte ao ciclo de vida de um OA. Neste projeto foram conduzidos experimentos objetivando 
(a) verificar a viabilidade da aplicação de ontologias na concepção/implementação de serviços, (b) comparar, quando possível, a qualidade dos resultados com outras soluções e (b) medir, o desempenho atingido com a implementação.

A metodologia de exploração tecnológica empregada no projeto OBAA-MILOS direcionou seus esforços primeiro para a busca de soluções de problemas particularmente difíceis ou complexos de aplicação de ontologias na representação e tratamento simbólico de OA. Assim, apesar de não ter sido realizada uma experimentação sistemática de todas as propriedades previstas no modelo ontológico delineado na Seção 8, com esses experimentos foram coletadas evidências significativas da viabilidade dessa abordagem. Os resultados apresentados em [Silva e Gluz, 2012; Gluz e Silva, 2014; Gluz et al., 2014a] mostram a viabilidade da aplicação de técnicas de alinhamento na fusão de ontologias educacionais. Esses experimentos também demonstram a viabilidade da utilização de mecanismos de mapeamento linguístico baseado em anotações para inter-relacionar as ontologias educacionais previamente alinhadas com a ontologia de metadados. Isso permitiu a criação de um serviço de busca semântica de OA efetivo e com boas evidências de qualidade e desempenho. Da mesma forma, experimentos realizados em [Silveira et al., 2013; Silveira e Gluz, 2012] mostram que técnicas de inferência baseadas em regras de produção tornam viável a utilização dos conhecimentos armazenados em ontologias educacionais, na criação/edição semiautomática de metadados de OA.

\section{Análise Comparativa}

O modelo ontológico proposto neste trabalho permite uma integração muito mais ampla entre os serviços do AVA e da plataforma semântica de conteúdos. Atualmente essa integração se dá principalmente através da disponibilização de OA empacotados em formato padrão SCORM ou IMS-CP [Penteado et al, 2014]. O suporte a outras atividades pedagógicas importantes como: (a) acompanhamento e avaliação das tarefas realizadas com o OA, (b) trabalho colaborativo e (c) autoaprendizado mediado pelo OA, se mostra muito limitado ou inexistente. O modelo definido na Seção 8 prevê ontologias específicas para cada uma dessas atividades, fornecendo os conhecimentos necessários para a implementação de serviços de suporte e apoio inteligente para cada uma dessas atividades.

Outra característica que se destaca na presente proposta é a própria integração dos serviços de suporte ao ciclo de vida de um OA em uma única plataforma semântica de gerência de conteúdos. Isso é um pouco incongruente devido a disponibilidade de uma variada gama de soluções para plataforma de gerência de conteúdos digitais como Joomla, Drupal, OpenCMS ou DotCMS, apenas se considerarmos plataformas opensource mais reconhecidas. Essa abordagem talvez seja devida ao fato de ambientes como o Moodle serem amplamente utilizados como plataformas de gerência e disponibilização de conteúdos educacionais. Independente disso, ao propor um modelo que define as propriedades e conhecimentos envolvidos em cada uma das atividades do ciclo de vida de um OA e definir claramente a interface dessa plataforma com os AVA (ver Figura 2) a presente proposta contribui para a evolução deste quadro de pesquisas.

\section{Referências}

Ball,S., Tenney, J. (2008) Xerte - A User-Friendly Tool for Creating Accessible Learning Objects. Lecture Notes in Computer Science 5105, pp. 291 a 294.

Barbone,V.G., Rifon,L.A. From SCORM to Common Cartridge: A step forward, 2009. Computers \& Education, 2010.88-102.

Bittencourt, I., Costa, E., Neto, B., Menezes, J., Melo, J., Ferneda, E., Silva, A. (2008) Constructing Intelligent Tutoring Systems Based on a Multiagent Architecture. In: Vicari, R. et al. (Org.). Agent-Based Tutoring Systems by Cognitive and Affective Modeling. 
Bittencourt, I., Costa, E., Silva, M., Soares, E. (2009) A computational model for developing semantic web-based educational systems. Knowledge-Based Systems, v. 22, n.4, p.302-315, $\mathrm{AI}$ in Blended Learning.

Ehrig, M. (2007) Ontology alignment: bridging the semantic gap. Springer.

Euzenat, J., Shvaiko, P. (2007) Ontology matching. Springer.

Gluz, J. C., Vicari, R. M. (2010) MILOS: Infraestrutura de Agentes para Suporte a Objetos de Aprendizagem OBAA. Anais SBIE 2010. João Pessoa.

Gluz, J.C., Vicari, R.M., Passerino, L.M. (2012) An Agent-Based Infrastructure for the Support of Learning Objects Life-Cycle . Procs. of ITS 2012, Chania, Crete. LNCS. New York: Springer, 2012. v.7315. 2012.

Gluz, J. C. ; Silva, L. R. J. (2014) Ontological semantic search of learning objects: experiments and results. Anais do SBIE 2014.

Gluz, J. C. ; Silva, L. R. J. ; Vicari, R. M. (2014) Aligning ontologies to bring semantics to learning object search. Proceedings of ITS 2014, Haway.

Gluz, J. C., Penteado, F., Mossmann, M., Gomes, L., Vicari, R. M. (2014) A Student Model for Teaching Natural Deduction based on a Prover that Mimics Student Reasoning. I Proceedings of ITS 2014. Haway.

Gomes, S. R., Gadelha, B.F. Castro, A. N. (2009) Objetos de Aprendizagem Funcionais: Uma Abordagem Prática. Anais do SBIE 2009, Florianópolis.

Hayashi, Y., Isotani, S., Bourdeau, J., Mizoguchi, R. (2009) Toward a Learning/Instruction Process Model for Facilitating the Instructional Design Cycle. Procs. of WCCE 2009.

Isotani, S., Mizoguchi, R., Bittencourt, I., Costa, E. (2008) Web 3.0 - Os Rumos da Web Semântica e da Web 2.0 nos Ambientes Educacionais. Anais do SBIE 2008. Fortaleza.

Isotani, S., Isotani, N., Isotani, S. (2008) Ontologias e Web Semântica no Suporte ao Ensino Colaborativo em Salas de Aula Presenciais. Anais do SBIE 2008. Fortaleza.

Jonassen, D. H. (1999) Learning with technology: a constructive perspective. USA: Prent. Hall.

Mizoguchi, R., Hayashi, Y., Bourdeau, J. (2007) Inside Theory-Aware \& Standards-Compliant Authoring System, Proceedings of SWEL'07, p.1-8.

Novo Dicionário Aurélio 3a edição (2004), Ed. Positivo.

Penteado, F., Gluz, J. C., Galafassi, C. (2014) Análise Crítica das Pesquisas Recentes sobre as Tecnologias de Objetos de Aprendizagem e Ambientes Virtuais de Aprendizagem. Revista Brasileira de Informática na Educação, v. 21, p. 41-52, 2014.

Schreiner, P., Rossi, L., Nunes, M., Viccari, R. (2012) FEB + OBAA: Cataloging and retrieving Learning Objects effectively. In: Applications to Provide Learning and Teaching Support Workshop, Anais dos Workshops do CBIE 2012. Rio de Janeiro.

Silva, M., Barros, H., Veras, D., Pacca, H., Ibert, I., Barros. (2009) Modelando um Sistema Educacional de MMC sob a perspectiva da Web Semântica. Anais SBIE 2009. Florianópolis.

Silva, L.J., Gluz, J. C. (2012) MSSearch: Busca Semântica de Objetos de Aprendizagem OBAA com Suporte a Alinhamento Automático de Ontologias. Anais SBIE 2012. Rio de Janeiro.

Silveira, E., Galão, M. C., Gluz, J. C. (2013) Uma ferramenta para fornecer apoio a catalogação de metadados de objetos de aprendizagem - LINNAEUS. Anais SBIE 2013. Campinas.

Silveira, E., Gluz, J. C. (2012) Sistema LINNAEUS: apoio inteligente para a catalogação e edição de metadados de objetos de aprendizagem. Anais do SBIE 2012. Rio de Janeiro.

Smith, M., Bass,M., Mcclellan, G., Tansley, R., Barton, M., Branschofsky, M., Stuve, D., Walker, J.H. (2003) DSpace An Open Source Dynamic Digital Repository, D-LIB Magazine.

Tomasello, M. (2001) The Cultural Origins of Human Cognition. Harvard University Press.

Vygotsky, L. S. (1998) Formação Social da Mente. $6^{\circ}$ Edição.- São Paulo: Martins Fontes.

Wertsch, J. (1998) Mind as Action. Oxford University Press.

Wiley, D. A. (2001) Connecting Learning Objects to Instructional Design Theory: A Definition, A Metaphor, and A Taxonomy.

Yu, E.. Giorgini, P., Maiden, N., Mylopoulos. J. Eds, (2001) Social Modeling for Requirements Engineering. The MIT Press, Cambridge, Massachusetts . 